\title{
Exts and Vertex Operators
}

\author{
Erik Carlsson and Andrei Okounkov
}

\begin{abstract}
The direct product of two Hilbert schemes of the same surface has natural K-theory classes given by the alternating Ext groups between the two ideal sheaves in question, twisted by a line bundle. We express the Chern classes of these virtual bundles in terms of Nakajima operators.
\end{abstract}

\section{Introduction}

\section{1}

Let $S$ be a nonsingular quasi-projective surface. The Hilbert scheme $\operatorname{Hilb}_{n} S$ of $n$ points on $S$ has been the focus of numerous recent studies, see e.g. [9, 13, 19] for a survey. In particular, the cohomology of $\mathrm{Hilb}_{n} S$ has been described using certain operators acting on

$$
\mathcal{F}=\bigoplus_{n} \mathcal{F}_{n}, \quad \mathcal{F}_{n}=H^{*}\left(\operatorname{Hilb}_{n} S, \mathbb{Q}\right)
$$

introduced by Nakajima [18] and Grojnowski [10].

In this paper we consider another natural set of operators, $\mathrm{W}(\mathcal{L})$, depending on a line bundle $\mathcal{L}$ on $S$. These operators act on $\mathcal{F}$, and are defined in terms of Chern classes of sheaves of Ext-groups. We prove an explicit formula for $W(\mathcal{L})$ in terms of the Nakajima operators, describing $W(\mathcal{L})$ as a vertex operator acting on $\mathcal{F}$.

Similar operators may be defined and studied for more general moduli spaces of sheaves. In this paper we will be content with the case of rank-one 
torsion-free sheaves on a surface (i.e. the Hilbert schemes of points), leaving generalizations to future papers.

The main application we have in mind concerns the case of $T$-equivariant cohomology of $\mathrm{Hilb}_{n} \mathbb{C}^{2}$, with respect to the natural action of $T \cong\left(\mathbb{C}^{*}\right)^{2}$ on $\mathbb{C}^{2}$. In this case, the trace of $\mathbf{W}(\mathcal{L})$ becomes one of Nekrasov's instanton partition functions, specifically the one with matter in the adjoint representation. Equivariant localization translates our formula into a rather surprising Pieri-type formula for Jack symmetric functions.

\section{2}

Let $S$ be a nonsingular surface and $\mathcal{L}$ a line bundle on $S$. The Hilbert scheme $\mathrm{Hilb}_{n}=\mathrm{Hilb}_{n} S$ parametrizes length $n$ subschemes $Z \subset S$ or, equivalently, ideal sheaves

$$
I=\operatorname{Ker}\left(\mathcal{O}_{S} \rightarrow \mathcal{O}_{Z}\right)
$$

of colength $n$. It comes with a universal subscheme

$$
\mathfrak{Z} \subset \operatorname{Hilb}_{n} S \times S
$$

whose fiber over $Z \in \mathrm{Hilb}_{n}$ is $Z$ itself. Consider

$$
\mathcal{Z}_{i}=p_{i 3}^{*}\left(\mathcal{O}_{\mathfrak{Z}}\right) \in K\left(\operatorname{Hilb}_{k} S \times \operatorname{Hilb}_{l} S \times S\right), \quad i=1,2,
$$

where, for example, $p_{13}$ denotes the projection to the first and third factors. The main object of this paper is the following class

$$
\mathrm{E}=p_{12_{*}}\left(\left(z_{1}^{\vee}+z_{2}-z_{1}^{\vee} \cdot z_{2}\right) \cdot p_{3}^{*}(\mathcal{L})\right) \in K\left(\operatorname{Hilb}_{k} \times \mathrm{Hilb}_{l}\right),
$$

where checks denote $K$-theoretic duals. Note that the fibers of $p_{3}$ intersect the supports of $z_{i}$ in finite sets, hence $p_{12 *}$ is well-defined without assuming $S$ is proper.

If $S$ is proper, then

$$
\left.\mathrm{E}\right|_{\left(I_{1}, I_{2}\right)}=\chi(\mathcal{L})-\chi\left(I_{1}, I_{2} \otimes \mathcal{L}\right)
$$

where

$$
\chi(F, G)=\sum_{i=0}^{2}(-1)^{i} \operatorname{Ext}^{i}(F, G)
$$


for a pair of coherent sheaves $F$ and $G$ on $S$. The subtraction in (2) is also meaningful if $I_{1}$ and $I_{2}$ are invariant under the action of a torus $T \subset \operatorname{Aut}(S)$ such that $S^{T}$ is proper. In that case, the Ext-groups have finite dimensional $T$-eigenspaces, and their difference is finite-dimensional, making (2) welldefined in $K_{T}(p t)$. For a reference on $K$-theory, especially the equivariant case, see [5]. For reference on equivariant cohomology in algebraic geometry, see Fulton's notes [8].

\section{3}

We have

$$
\operatorname{rk} \mathrm{E}=k+l
$$

and we denote

$$
e(\mathrm{E})=c_{k+l}(\mathrm{E}) .
$$

We define the operator $\mathrm{W}(\mathcal{L})=\mathrm{W}(\mathcal{L}, z) \in \operatorname{End}(\mathcal{F})\left[\left[z, z^{-1}\right]\right]$ by its matrix elements

$$
(\mathrm{W}(\mathcal{L}) \eta, \xi)=z^{l-k} \int_{\mathrm{Hilb}_{k} \times \operatorname{Hilb}_{l}} p_{1}^{*}(\eta) \cup p_{2}^{*}(\xi) \cup e(\mathrm{E})
$$

where

$$
\eta \in H_{T}^{*}\left(\operatorname{Hilb}_{k}\right), \quad \xi \in H_{T}^{*}\left(\operatorname{Hilb}_{l}\right)
$$

and

$$
(\eta, \xi)=\int \eta \cup \xi
$$

Here and in what follows integration is defined by equivariant localization:

$$
\int_{X} \eta=\sum_{a} \int_{F_{a}} \frac{\iota_{a}^{*} \eta}{e_{a}} \in \mathbb{C}\left(\mathfrak{t}^{*}\right), \quad \mathfrak{t}=\operatorname{Lie}(T),
$$

where

$$
\iota_{a}: F_{a} \rightarrow X
$$

is the inclusion of a connected component of $X^{T}$ and $e_{a}$ is the Euler class of its normal bundle. The fixed-point loci will be always assumed proper, so $\int_{F_{a}}$ in (4) is the usual nonequivariant integral. The localization formula states that the integrals as defined in (4) commute with proper equivariant pushforwards, see [11] for a reference.

For each $v \in \mathcal{F}, \mathbf{W}(\mathcal{L}) \cdot v$ is a formal power series in $z, z^{-1}$ with coefficients in $\mathcal{F}$ such that that coefficient of $z^{N}$ is 0 for $N \ll 0$. 


\section{4}

Note that one can always twist $\mathcal{L}$ by a torus character, even when the torus action on $S$ is trivial. The result is a scalar torus action on the fibers of $\mathrm{E}$ and hence $e(\mathrm{E})$, as $T$-equivariant class, effectively includes all smaller Chern classes of $E$. As we will see,

$$
c_{i}(\mathrm{E})=0, \quad i>k+l .
$$

Note also that by Serre duality,

$$
\chi(I, J \otimes \mathcal{L})=\chi\left(J, I \otimes \mathcal{K} \otimes \mathcal{L}^{-1}\right),
$$

so that

$$
\mathrm{W}(\mathcal{L}, z)^{*}=(-1)^{\mathrm{N}} \mathrm{W}\left(\mathcal{K}-\mathcal{L}, z^{-1}\right)(-1)^{\mathrm{N}},
$$

where $\mathcal{K}$ is the canonical bundle of $S$, and $\mathrm{N}$ is the number-of-points operator,

$$
\left.\mathrm{N}\right|_{\mathcal{F}_{n}}=n \mathrm{Id} .
$$

\section{5}

Our goal is to relate the operator $\mathrm{W}(\mathcal{L})$ to Nakajima operators on $\mathcal{F}$ which are defined as follows. For $k<l$ define a cycle by

$$
\mathfrak{A}=\{(I, x, J) \mid J \subset I, \operatorname{supp}(J / I)=\{x\}\} \subset \operatorname{Hilb}_{k} S \times S \times \operatorname{Hilb}_{l} S .
$$

For each $\gamma \in H_{*}^{T}(S)$ define an operator by

$$
\left(\alpha_{k-l}(\gamma) \eta, \xi\right)=\int p_{1}^{*}(\eta) \cup p_{3}^{*}(\xi) \cap p_{2}^{*}(\gamma) \cap[\mathfrak{A}], \quad \eta \in \mathcal{F}_{k}, \xi \in \mathcal{F}_{l}
$$

and let $\alpha_{n}(\gamma)=\alpha_{-n}(\gamma)^{*}$ for $n>0$. We will use the shorthand $\alpha_{-n}(\mathcal{L})$ for the case when $\gamma$ is the Poincaré dual of $c_{1}(\mathcal{L})$. Nakajima's theorem says that

$$
\left[\alpha_{i}(\gamma), \alpha_{j}\left(\gamma^{\prime}\right)\right]=(-1)^{i-1} i \delta_{i,-j}\left(\gamma, \gamma^{\prime}\right)_{S},
$$

where the brackets denote the supercommutator with respect to the standard $\mathbb{Z} / 2$-grading in cohomology.

Now we can state our main result 


\section{Theorem 1.}

$$
\mathbf{W}(\mathcal{L})=\exp \left(\sum_{n>0} \frac{(-1)^{n-1} z^{n}}{n} \alpha_{-n}(\mathcal{L})\right) \exp \left(-\sum_{n>0} \frac{z^{-n}}{n} \alpha_{n}(\mathcal{K}-\mathcal{L})\right) .
$$

In particular, one can take $k=0$ in (3). The second factor in (66) fixes the vacuum vector

$$
|\rangle=1 \in H^{*}\left(\mathrm{Hilb}_{0}\right) \subset \mathcal{F},
$$

while $\mathrm{E}$ becomes the tautological bundle $\chi((\mathcal{O} / J) \otimes \mathcal{L})$. In this case, (6) specializes to a formula of M. Lehn, see corollary 6.6 in [13].

The product in (6) is a vertex operator in the sense that it may be uniquely characterized by its commutation relations with the Heisenberg algebra spanned by the Nakajima operators. It would be very interesting to find a geometric reason for this. Various connections and parallels between Hilbert schemes and vertex operator algebras may be found in the work of Li, Qin, and Wang, see e.g. [14].

\section{Corollary 1.}

$$
\operatorname{str} q^{\mathrm{N}} W(\mathcal{L})=\prod_{n}\left(1-q^{n}\right)^{(\mathcal{L}, \mathcal{K}-\mathcal{L})-e(S)}
$$

where the right hand side is the Taylor series about $q=0$, and $e(S)$ is the Euler characteristic of $S$.

This will be deduced from the main theorem in Section 2.2

\section{6}

Parallel structures exist in the $K$-theory of Hilbert schemes, see [4]. The authors would like to thank Davesh Maulik, Nikita Nekrasov, and the referees.

\section{Applications}

\subsection{Nekrasov theory}

\subsection{1}

If $\mathcal{L}=\mathcal{O}$ then

$$
\left.\mathrm{E}\right|_{\text {diagonal in } \operatorname{Hilb}_{n} \times \operatorname{Hilb}_{n}}=T \operatorname{Hilb}_{n}
$$


in $K$-theory, see Proposition 2.2 in [7]. This explains why

$$
\operatorname{str} q^{\mathrm{N}} W(\mathcal{O})=\prod_{n}\left(1-q^{n}\right)^{-e(S)},
$$

agrees with the generating functions for $e\left(\mathrm{Hilb}_{n}\right)$ obtained from Göttsche's formula [9].

Twisting $\mathcal{O}$ by a torus character $m$, we get the Chern polynomial of the tangent bundle. Hence

$$
\operatorname{str} q^{\mathrm{N}} W(\mathcal{O}(m))=\sum_{n} q^{n} \int_{\mathrm{Hilb}_{n}} c\left(T \mathrm{Hilb}_{n}, m\right)
$$

By definition, this series is the instanton part of Nekrasov partition function with for the $U(1)$-adjoint matter of mass $m$, a subject of considerable physical interest, see for example [20]. In particular, corollary 1 generalizes formula (6.12) in [20]. More generally, we have the following

Corollary 2. For any $\phi \in \mathcal{F}$,

$$
\sum_{n} q^{n} \int_{\mathrm{Hilb}_{n}} \phi \cup c\left(T \operatorname{Hilb}_{n}, m\right)=\operatorname{str} q^{\mathrm{N}} \Phi W(\mathcal{O}(m)),
$$

where $\Phi: \mathcal{F} \rightarrow \mathcal{F}$ is the operator of multiplication by $\phi$ in the cohomology of $\mathrm{Hilb}_{n}$.

\subsection{2}

Series of the form (77) are correlation functions in Nekrasov theory. They are studied in detail in [3]. Generalizing (7), we denote

$$
\langle\phi\rangle=\operatorname{str} q^{\mathrm{N}} \Phi W(\mathcal{L})=\left.\sum_{n} q^{n} \int_{\mathrm{Hilb}_{n} S} \phi \cup e(\mathrm{E})\right|_{\text {diagonal }} .
$$

Corollary 1 is the computation of $\langle 1\rangle$. The next simplest correlator is computed in the following

Corollary 3. Let $\phi=c_{1}(\mathcal{O} / \mathcal{J})$ be the 1 st Chern class of the tautological bundle $\mathcal{O} / \mathcal{J}$ on $\mathrm{Hilb}_{n} S$. Then

$$
\frac{\left\langle c_{1}(\mathcal{O} / \mathcal{J})\right\rangle}{\langle 1\rangle}=\frac{1}{2}\left(E_{2}(q)-E_{3}(q)\right) \int_{S}\left(c_{1} c_{2}-c_{3}\right)(T S \oplus \mathcal{L}) .
$$


Here the series

$$
E_{k}(q)=\sum_{n>0} n^{k-1} \frac{q^{n}}{1-q^{n}}
$$

is a $q$-analog of $\zeta(k)$ and also an $S L_{2}(\mathbb{Z})$-Eisenstein series without constant term for $k$ positive even.

The characteristic class $c_{1} c_{2}-c_{3}$ in (8) refers to the rank 3 bundle $T S \oplus \mathcal{L}$ on the surface $S$. Its integral over $S$ has degree 1 in equivariant parameters.

\subsubsection{Chern classes of $T$ Hilb}

Corollary 2 gives a procedure to compute the Chern classes of the tangent bundle to the Hilbert scheme $\mathrm{Hilb}_{n} S$ in any basis $\left\{\phi_{i}\right\}$ of the cohomology, such as the Nakajima basis. To use it, one has to identify the corresponding operators $\left\{\Phi_{i}\right\}$ of multiplication by $\phi_{i}$ in cohomology.

One algorithm for producing the multiplication operators $\Phi_{i}$ is discussed in [13]. There are also other approaches, see for example [14, 17].

\subsection{Proof of corollaries 1 and 3}

\subsection{1}

Write Theorem 11 as $W(\mathcal{L})=\Gamma_{-}(-\mathcal{L},-z) \Gamma_{+}(\mathcal{L}-\mathcal{K}, z)$, where

$$
\Gamma_{ \pm}(\mathcal{L}, z)=\exp \left(\sum_{n>0} \frac{z^{\mp n} \alpha_{ \pm n}(\mathcal{L})}{n}\right) .
$$

Using Nakajima's commutation relations,

$$
\begin{aligned}
\operatorname{str} q^{\mathrm{N}} & \Gamma_{-}(-\mathcal{L},-z) \Gamma_{+}(\mathcal{L}-\mathcal{K}, z) \\
& =\operatorname{str} q^{\mathrm{N}} \Gamma_{+}(\mathcal{L}-\mathcal{K}, z) \Gamma_{-}(-\mathcal{L},-z q) \\
& =(1-q)^{(\mathcal{L}, \mathcal{K}-\mathcal{L})} \operatorname{str} q^{\mathrm{N}} \Gamma_{-}(-\mathcal{L},-z q) \Gamma_{+}(\mathcal{L}-\mathcal{K}, z) \\
& =\prod_{n}\left(1-q^{n}\right)^{(\mathcal{L}, \mathcal{K}-\mathcal{L})} \operatorname{str} q^{\mathrm{N}} \Gamma_{+}(\mathcal{L}-\mathcal{K}, z) .
\end{aligned}
$$

The trace of these operators is defined as a formal power series in $q$, which makes sense because each coefficient is an operator whose trace is a finite sum. We have used the rule that $\operatorname{str}(A B)=\operatorname{str}(B A)$ whenever $A$ and $B$ are operators which raise the degree by an even amount. Now corollary 1 follows from Göttsche's formula, and the fact that $\Gamma_{+}$is lower-triangular. 


\subsection{2}

By the reduction procedure used in the proof of Theorem 1, it suffices to prove (8) for a toric surface $S$. By localization, we can further reduce to $\mathbb{C}^{2}$. As in Section 3.7, we denote by $t_{1}, t_{2}$ the equivariant weights of $T \mathbb{C}^{2}$ and by $m$ the equivariant weight of $\mathcal{L}=\mathcal{O}_{\mathbb{C}^{2}}$. Then

$$
\int_{\mathbb{C}^{2}}\left(c_{1} c_{2}-c_{3}\right)\left(T \mathbb{C}^{2} \oplus \mathcal{L}\right)=\frac{\left(t_{1}+t_{2}\right)\left(t_{1}+m\right)\left(t_{2}+m\right)}{t_{1} t_{2}}
$$

On the other hand, the operator $\Phi$ for $\phi=c_{1}(\mathcal{O} / \mathcal{J})$ is given by a formula due to M. Lehn 12

$$
\Phi=-\frac{t_{1}+t_{2}}{2} \sum_{k \geq 1}(k-1) \alpha_{-k} \alpha_{k}+\sum_{k, l \geq 1} \frac{t_{1} t_{2}}{2} \alpha_{-k} \alpha_{-l} \alpha_{k+l}-\frac{1}{2} \alpha_{-k-l} \alpha_{k} \alpha_{l},
$$

in which we use the shorthand

$$
\alpha_{-n}=\alpha_{-n}(1), \quad \alpha_{n}=(-1)^{n-1} t_{1} t_{2} \alpha_{-n}^{*}
$$

so that $\left[\alpha_{m}, \alpha_{n}\right]=m \delta_{m,-n}$. Compare Lehn's commutation relations with the commutator of the above formula for $\Phi$ with $\alpha_{n}$.

We use the commutation relations

$$
\left[\alpha_{ \pm k}, \Gamma_{\mp}^{m}(z)\right]= \pm m z^{\mp k} \Gamma_{\mp}^{m}(z), \quad \Gamma_{ \pm}^{m}(z)=\exp \left(m \sum_{k} \frac{z^{\mp k}}{k} \alpha_{ \pm k}\right)
$$

repeatedly to move the vertex operators around the trace as in the proof of corollary 1 above. It is slightly simpler to use the dual of the vertex operator under the inner-product (22), because it eliminates some awkward signs. Since

$$
c_{1}(\mathcal{K})=-t_{1}-t_{2} \in \mathbb{C}\left[t_{1}, t_{2}\right], \quad c_{1}(\mathcal{L})=m
$$

we get

$$
W(m)^{*}=\Gamma_{-}^{m+t_{1}+t_{2}}(1) \Gamma_{+}^{m / t_{1} / t_{2}}(1) .
$$

Taking the trace against one of the terms in $\Phi$ gives, for instance,

$$
\begin{gathered}
\operatorname{tr} q^{N} \alpha_{-k-l} \alpha_{k} \alpha_{l} \Gamma_{-}^{m+t_{1}+t_{2}}(1) \Gamma_{+}^{m / t_{1} / t_{2}}(1)= \\
\frac{m\left(m+t_{1}+t_{2}\right)^{2}}{t_{1} t_{2}} \frac{q^{k+l}}{\left(1-q^{k}\right)\left(1-q^{l}\right)\left(1-q^{k+l}\right)}\langle 1\rangle .
\end{gathered}
$$


Combining all three terms in $\Phi$, we get

$$
\begin{aligned}
-\frac{\left\langle c_{1}(\mathcal{O} / \mathcal{J})\right\rangle}{\langle 1\rangle}= & \frac{t_{1}+t_{2}}{2} \sum_{k} k(k-1) \frac{q^{k}}{1-q^{k}}+ \\
& \frac{\left(t_{1}+t_{2}\right)\left(m+t_{1}+t_{2}\right) m}{2 t_{1} t_{2}} \sum_{k}(k-1) \frac{q^{k}}{\left(1-q^{k}\right)^{2}}+ \\
& \frac{\left(t_{1}+t_{2}\right)\left(m+t_{1}+t_{2}\right) m}{2 t_{1} t_{2}} \sum_{k, l} \frac{q^{k+l}}{\left(1-q^{k}\right)\left(1-q^{l}\right)\left(1-q^{k+l}\right)} .
\end{aligned}
$$

The first two $q$-series above are easily seen to equal $E_{3}-E_{2}$ and $q \frac{d}{d q} E_{1}-E_{2}$, respectively. The third is identified in the following

\section{Lemma 4.}

$$
\sum_{k, l>0} \frac{q^{k+l}}{\left(1-q^{k}\right)\left(1-q^{l}\right)\left(1-q^{k+l}\right)}=E_{3}-q \frac{d}{d q} E_{1} .
$$

Proof. We denote $[n]=q^{n / 2}-q^{-n / 2}$ and consider

$$
f(z)=\sum_{n>0} \frac{z^{n}+z^{-n}}{[n]}, \quad\left|q^{1 / 2}\right|<|z|<\left|q^{-1 / 2}\right| .
$$

We have

$$
\text { LHS }=-\frac{1}{6}\left[z^{0}\right] f(z)^{3}
$$

where $\left[z^{0}\right]$ denotes the coefficient of $z^{0}$. From

$$
\frac{1}{[n][n+l]}=\frac{1}{[l]}\left(\frac{q^{n / 2}}{[n]}-\frac{q^{(n+l) / 2}}{[n+l]}\right)
$$

we find, for $l \neq 0$ and $|q|<1$, the following series telescopes:

$$
\left[z^{l}\right] f(z)^{2}=\sum_{a+b=l, a b>0} \frac{1}{[a][b]}+\frac{2}{[l]} \sum_{a=1}^{|l|} \frac{q^{a / 2}}{[a]} .
$$

Comparing this to (11) and setting $\left(n_{1}, n_{2}\right)=(l, a)$, we find

$$
\text { LHS }=-\sum_{n_{1} \geq n_{2}>0} \frac{q^{n_{2} / 2}}{\left[n_{2}\right]\left[n_{1}\right]^{2}}
$$


Now (10) follows at once from the following analog

$$
\sum_{n_{1} \geq n_{2}>0} \frac{q^{-n_{2} / 2}}{\left[n_{2}\right]\left[n_{1}\right]^{2}}=\sum_{n>0} \frac{q^{n / 2}}{[n]^{3}}
$$

of Euler's $\zeta(2,1)=\zeta(3)$ identity proven by Bradley. Namely, (12) is the $k=n=2$ case of corollary 4 in [1].

One collects the terms in (9) to finish the proof of corollary 3 ,

\subsection{Pieri-type formulas for Jack polynomials}

Recall that algebra $\Lambda$ of symmetric functions over $\mathbb{Q}$ is freely generated by the power-sum symmetric functions $p_{k}$, where $k=1,2, \ldots$, that is,

$$
\Lambda=\mathbb{Q}\left[p_{1}, p_{2}, p_{3}, \ldots\right] .
$$

Consider the Jack inner product $(\cdot, \cdot)_{\theta}$ on $\Lambda$ with parameter $\theta$. This is the unique inner product such that

$$
p_{k}^{*}=\frac{k}{\theta} \frac{\partial}{\partial p_{k}}
$$

where $p_{k}$ is viewed as the corresponding multiplication operator. We have $\theta=1 / \alpha$, where $\alpha$ is the parameter used in Macdonald's book [16]. The integral form $J_{\mu}$ of Jack symmetric functions is obtained by Gram-Schmidt orthogonalization of monomial symmetric functions with respect to $(\cdot, \cdot)_{\theta}$. It is normalized so that the coefficient of $p_{1}^{|\mu|}$ in $J_{\mu}$ equals 1 .

Let $E$ be the operator of multiplication by the sum of all elementary symmetric functions

$$
E=1+e_{1}+e_{2}+\cdots=\exp \left(\sum_{n} \frac{(-1)^{n}}{n} p_{n}\right) .
$$

and $E^{*}$ the adjoint operator. Specialized to the equivariant cohomology of Hilb $_{n} \mathbb{C}^{2}$, formula (6) may be restated as the following Pieri-type formula for Jack polynomials:

\section{Corollary 5.}

$$
\begin{aligned}
& \left(E^{m}\left(E^{*}\right)^{\theta-m-1} J_{\lambda}, J_{\mu}\right)_{\theta}=(-1)^{|\lambda|} \theta^{-|\lambda|-|\mu|} \times \\
& \quad \prod_{\square \in \lambda}\left(m+a_{\lambda}(\square)+1+\theta l_{\mu}(\square)\right) \prod_{\square \in \mu}\left(m-a_{\mu}(\square)-\theta\left(l_{\lambda}(\square)+1\right)\right) .
\end{aligned}
$$


We note that in the product (13) the ranges $\square \in \lambda$ and $\square \in \mu$ may be interchanged, see the proof of Lemma 6 below.

\section{Proof of Theorem 1}

\section{1}

Let $\Gamma(\mathcal{L})$ denote the right-hand side of $(\underline{6})$ so

$$
\mathrm{W}(\mathcal{L})=\Gamma(\mathcal{L})
$$

is what we need to prove. As a first step, we reduce the general formula (14) to the special case of the equivariant cohomology of a toric surface $S$.

Consider the universal ideal sheaf $\mathcal{J}$ on $\operatorname{Hilb}_{k} S \times S$ and the Künneth components of its Chern character

$$
\sigma_{i}(\gamma)=\int_{S} \operatorname{ch}_{i+2}(\mathcal{J}) \gamma \quad \in H^{2 i+\operatorname{deg} \gamma}\left(\operatorname{Hilb}_{k}\right)
$$

It is known that these classes generate $H^{*}\left(\mathrm{Hilb}_{k}\right)$ as a ring, that is, the monomials in these classes span $H^{*}\left(\mathrm{Hilb}_{k}\right)$, see e.g. [14] and Section 6 in [13].

For given $k$ and $l$, consider a matrix coefficient of $\mathrm{W}(\mathcal{L})$ between two such monomials

$$
\left(\mathrm{W}(\mathcal{L}) \prod \sigma_{p_{i}}\left(\eta_{i}\right), \prod \sigma_{q_{j}}\left(\xi_{j}\right)\right)
$$

We may assume that the cohomology classes $\eta_{i}$ and $\xi_{j}$ have a well-defined parity.

Using the Grothendieck-Riemann-Roch formula, we may express (15) as an integral over

$$
\operatorname{Hilb}_{k} S \times \operatorname{Hilb}_{l} S \times S \times S \times \cdots \times S
$$

of a universal expression in characteristic classes of

$$
\pi_{r s}^{*} \mathcal{J}, \quad r=1,2 \quad s \geq 3 \ldots,
$$

where $\pi_{r s}$ is the projection on the $r$ th and sth factor in (16), times a class $\gamma_{s}$ of the form

$$
\gamma_{s} \in\left\{\eta_{i}, \xi_{j}, \operatorname{ch}_{k}(\mathcal{L}) \operatorname{td}_{k}(S)\right\}
$$

for each of the $S$-factors in (16). 
The induction scheme of Ellingsrud, Göttsche, and Lehn, see Section 3 in [7, gives a universal evaluation of any such integral. The result has the form

$$
\sum_{\{3,4, \ldots\}=\sqcup G_{i}} \pm \prod_{i} \int_{S} C_{G, i} \prod_{s \in G_{i}} \gamma_{s}
$$

where the outer sum is over all partitions of the $S$-factors in (16) into disjoint groups $G_{i}$, the sign accounts for a possibly different ordering of odd cohomology classes in the original and final integral, and each $C_{G, i} \in H^{*}(S)$ is a universal polynomial, independent of the $\gamma_{i} s$, applied to the characteristic classes of the tangent bundle of $S$.

Next, we show that the matrix coefficients of $\Gamma(\mathcal{L})$ are given by a similar universal formula. To do this, we use the calculus explained in Section 6 of [13], and subsequent generalizations in [14] to express the operator of the cup product with $\sigma_{p}(\eta)$ in terms of suitable Nakajima operators. We may then express the classes $\prod \sigma_{p_{i}}\left(\eta_{i}\right)$ in terms of operators of the form

$$
\alpha_{\lambda}\left(\Delta_{\ell *} \gamma\right)=\sum_{j} \alpha_{\lambda_{1}}(\gamma(j, 1)) \cdots \alpha_{\lambda_{\ell}}(\gamma(j, \ell))
$$

applied to the vacuum. Here $\lambda$ is a generalized partition, $\ell$ is the length of $\lambda, \Delta_{\ell}$ is the diagonal embedding, $\gamma$ is the product of $\eta_{i}$ with a characteristic class of $S$, and $\gamma(j, i)$ are the Künneth components of the diagonal, $\Delta_{\ell *} \gamma=$ $\sum_{j} \gamma(j, 1) \otimes \cdots \otimes \gamma(j, \ell)$.

Expanding $\Gamma(\mathcal{L})$, it suffices to show that

$$
\left\langle\left|\alpha_{\lambda^{(1)}}\left(\Delta_{\ell_{1} *} \gamma_{1}\right) \cdots \alpha_{\lambda^{(k)}}\left(\Delta_{\ell_{k} *} \gamma_{k}\right)\right|\right\rangle
$$

is given by an expression of the form (17) with $\gamma_{s} \in\left\{\gamma_{i}\right\}$. Expanding this expression, and using the Heisenberg relations gives

$$
\begin{aligned}
& \left\langle\left|\alpha_{\lambda^{(1)}}\left(\Delta_{\ell_{1} *} \gamma_{1}\right) \cdots \alpha_{\lambda^{(k)}}\left(\Delta_{\ell_{k} *} \gamma_{k}\right)\right|\right\rangle= \\
& \sum_{j_{1}, \ldots, j_{k}} \sum_{X} C(X) \prod_{(x, y) \in X} \int_{S} \gamma_{x_{1}}\left(j_{x_{1}}, x_{2}\right) \cdot \gamma_{y_{1}}\left(j_{y_{1}}, y_{2}\right)= \\
& \sum_{X} C(X) \int_{S \times \cdots \times S} \gamma_{1} \otimes \cdots \otimes \gamma_{k} \cdot f_{X}\left(p_{i j}^{*} \mathcal{O}_{\Delta}\right),
\end{aligned}
$$

where $X$ is a perfect matching of the set

$$
\left\{(a, b), 1 \leq a \leq k, 1 \leq b \leq l_{a}\right\}
$$


$C(X)$ are numbers, and $f_{X}$ is some polynomial depending on $X$. This is of the desired form.

We now show that it suffices to check the equality of $W(\mathcal{L})$ and $\Gamma(\mathcal{L})$ when $S$ is a toric variety. First notice that grouping the $\operatorname{ch}_{k}(\mathcal{L}) \operatorname{td}_{k}(S)$ with the universal polynomials $C_{G, i}$ gives an alternative expression of the same form as (17), but where $\gamma_{s}$ varies over the $\left\{\eta_{i}, \xi_{j}\right\}, C_{G, i}$ is a universal polynomial in $\operatorname{ch}_{k}(\mathcal{L})$ and the characteristic classes of $S$, and $G$ partitions the set $\{1, \ldots, d\}$, where $d$ is the number of classes $\eta_{i}, \xi_{j}$.

Now suppose $W(\mathcal{L})$ agrees with $\Gamma(\mathcal{L})$ whenever $S$ is toric, but not for some nontoric $S$. Then there exists a universal family of polynomials $C_{G, i}$ as in the previous paragraph, such that (17) vanishes as an equivariant integral whenever $S$ is a toric variety, but not on some nontoric variety. Let $C$ be such a collection, and let $G$ be a partition such that $C_{G, i}$ is not the zero polynomial for any $i$, and no proper refinement of $G$ also has this property. If $S$ is a toric variety with at least $d$ fixed points $p_{s}$, let

$$
\gamma_{s}=\sum_{t \in G_{i}}\left[p_{t}\right]
$$

where $i$ is such that $s \in G_{i}$, and $\left[p_{s}\right]$ is the class of the fixed point $p_{s}$. These classes have the property that $\prod_{s} \gamma_{s}$ is nonzero when $s$ varies within some $G_{i}$, but zero otherwise. It is simple to find toric $S, \mathcal{L}$, such that

$$
\prod_{i} \int_{S} C_{G, i} \prod_{s \in G_{i}} \gamma_{s}
$$

is nonzero. But by the choice of $G$ and $\gamma_{s}$, this is the only contributing term in (17), which is assumed to vanish.

\section{2}

Let $T_{0}=U(1) \times \cdots \times U(1) \subset T$ be the compact torus with Lie algebra $\mathfrak{t}_{0}$. We have $S^{T_{0}}=S^{T}$ and since we now assume $S$ toric this is a finite set. Let $\left\{U_{s}\right\}$ be disjoint $T_{0}$-invariant open neighborhoods of the fixed points $s \in S$. By equivariant localization and the obvious inclusion map

$$
\bigsqcup_{s} \operatorname{Hilb}_{n_{s}} U_{s} \hookrightarrow \operatorname{Hilb}_{n} S, \quad n=\sum_{s} n_{s}
$$


we obtain an isomorphism

$$
\mathcal{F}_{S} \otimes_{\mathbb{C}\left[\mathfrak{t}^{*}\right]} \mathbb{C}\left(\mathfrak{t}^{*}\right)=\bigotimes_{s \in S^{T}} \mathcal{F}_{U_{s}} \otimes_{\mathbb{C}\left[\mathfrak{t}^{*}\right]} \mathbb{C}\left(\mathfrak{t}^{*}\right)
$$

Here each factor

$$
\mathcal{F}_{U_{s}} \cong \mathcal{F}_{T_{s} S}
$$

corresponds to the Hilbert scheme of points of $\mathbb{C}^{2} \cong T_{s} S$ with the inherited torus action.

In (19), we have an obvious factorization

$$
\mathrm{W}_{S}(\mathcal{L})=\bigotimes_{s \in S^{T}} \mathrm{~W}_{T_{s} S}\left(\left.\mathcal{L}\right|_{s}\right)
$$

Furthermore, $\Gamma(\mathcal{L})$ factors similarly, since

$$
j^{*}([\mathfrak{A}(S)])=[\mathfrak{A}(U)],
$$

where $j$ is the induced from the inclusion of an open neighborhood $U \subset S$, and $j^{*}$ is the restriction map on Borel-Moore homology. See [2] or [6] for a reference on equivariant Borel-Moore cohomology and intersection theory. The corresponding decomposition

$$
\alpha_{-n}(\gamma)=\bigoplus_{s} \alpha_{-n}\left(\left.\gamma\right|_{s}\right)
$$

leads to the desired factorization of $\Gamma(\mathcal{L})$, which reduces our theorem to the case $S=\mathbb{C}^{2}$. For $S=\mathbb{C}^{2}$, the only line bundles are torus characters.

\section{3}

The Fock space $\mathcal{F}_{\mathbb{C}^{2}}$ may be identified with symmetric functions in such a way that Nakajima operators become the operators of multiplication by the power-sum symmetric functions, while the classes of torus-fixed points are mapped to properly normalized Jack symmetric functions. Since the character of the torus action in the fiber of $E$ over any fixed point is easily determined (see below), the statement of the theorem may be rephrased purely as a statement about symmetric functions.

Instead of attacking the symmetric function problem directly, we will first use geometric arguments to reduce it further to a simple special case. 


\section{4}

Consider the following setup:

$$
S=\mathbf{P}^{1} \times \mathbf{P}^{1}, \quad T=\mathbb{C}^{*} \times \mathbb{C}^{*} \times \mathbb{C}^{*}, \quad \mathcal{L}=\mathcal{O}(m),
$$

where $\left(z_{1}, z_{2}, z_{3}\right) \cdot(x, y)=\left(z_{1} x, z_{2} y\right)$, and $z_{3}=e^{m}$ acts by scaling $\mathcal{L}$.

Take two partitions $\mu$ and $\nu$ such that $|\mu|=k$ and $|\nu|=l$ and consider

$$
w_{\mu, \nu}=w_{S}(\mu, \nu)=\left(\mathrm{W}(\mathcal{L}, 1) \prod \alpha_{-\mu_{i}}\left(L_{1}\right)|\rangle, \prod \alpha_{-\nu_{i}}\left(L_{2}\right)|\rangle\right) \in \mathbb{Z},
$$

where

$$
L_{1}=\{\mathrm{pt}\} \times \mathbf{P}^{1}, \quad L_{2}=\mathbf{P}^{1} \times\{\mathrm{pt}\} .
$$

Let also $w_{\mathbb{C}^{2}}^{[a b]}(\mu, \nu), a, b \in\{0, \infty\}$, be the same expression over the Hilbert scheme of the chart of $\mathbf{P}^{1} \times \mathbf{P}^{1}$ by $\mathbb{C}^{2}$ that contains the point $(a, b)$. Use the intersection of $L_{1}, L_{2}$ with $\mathbb{C}^{2}$ in place of $L_{1}, L_{2}$.

Since $w_{\mu, \nu}$ is an integral of a top-dimensional class over a complete space, it is independent of the equivariant parameters (including $m$ ) as well as the choice of the equivariant lifts of the classes $L_{1}$ and $L_{2}$. Similarly, the number

$$
g_{\mu, \nu}=\left(\Gamma(\mathcal{L}, 1) \prod \alpha_{-\mu_{i}}\left(L_{1}\right)|\rangle, \prod \alpha_{-\nu_{i}}\left(L_{2}\right)|\rangle\right)
$$

may be computed in either equivariant or ordinary cohomology and, in particular, is independent of equivariant parameters. We can use this independence as follows.

\section{5}

Let us compute $w_{\mu, \nu}$ using equivariant localization. Each occurrence of the line $L_{1}$ may be lifted to two different classes in equivariant cohomology, namely,

$$
L_{1}^{0}=\{0\} \times \mathbf{P}^{1} \quad \text { or } \quad L_{1}^{\infty}=\{\infty\} \times \mathbf{P}^{1},
$$

and similarly for $L_{2}$. Such a lifting corresponds to a decomposition

$$
\mu=\mu^{[0]} \sqcup \mu^{[\infty]}, \quad \nu=\nu^{[0]} \sqcup \nu^{[\infty]} .
$$

Any choice of lifting allows us to compute $w, g$ by localization, so the answer must be independent of this lifting. In particular, using the above factorization of $\mathrm{W}(\mathcal{L})$,

$$
w_{\mu, \nu}=\sum_{\mu^{[00], \mu}[0 \infty], \mu\left[\infty{ }^{[\infty], \mu}[\infty \infty]\right.} \sum_{\nu^{[00], \nu}[0 \infty], \nu[\infty], \nu} \prod_{\nu^{[\infty \infty}} w_{a, b}^{[a b]}\left(\mu^{[a b]}, \nu^{[a b]}\right),
$$


where $a, b \in\{0, \infty\}$, and the sum is over terms such that $\mu^{[a 0]} \sqcup \mu^{[a \infty]}=$ $\mu^{[a]}, \nu^{[0 b]} \sqcup \nu^{[\infty b]}=\nu^{[b]}$, for any fixed $\mu^{[0]}, \mu^{[\infty]}, \nu^{[0]}, \nu^{[\infty]}$. Using the parallel factorization for $\Gamma$, (21) also holds when $\mathrm{W}$ is replaced with $\Gamma$, and $w$ is replaced with $g$.

Equation (21) is a collection of relations for each decomposition of $\mu$ and $\nu$. These formulas for both $w$ and $g$ give an induction step for proving that $w_{\mathbb{C}^{2}}=g_{\mathbb{C}^{2}}$, which is sufficient to prove the theorem. First, setting $\mu^{[a]}=\mu$, $\nu^{[b]}=\nu$, and solving for $w_{\mathbb{C}^{2}}^{[a b]}$, we get

$$
w_{\mathbb{C}^{2}}^{[a b]}(\mu, \nu)=w_{S}(\mu, \nu)-\ldots
$$

where the remaining part of the sum consists of lower order terms. In other words, they involve $w_{\mathbb{C}^{2}}^{[c d]}\left(\mu^{\prime}, \nu^{\prime}\right)$ with $\ell\left(\mu^{\prime}\right) \leq \ell(\mu), \ell(\nu)^{\prime} \leq \ell(\nu)$, and either $\ell\left(\mu^{\prime}\right)<\ell(\mu)$ or $\ell\left(\nu^{\prime}\right)<\ell(\nu)$. If $\ell(\mu)>1$ or $\ell(\nu)>1$, then using any decomposition of $\mu$ or $\nu$ into strictly smaller components yields $w_{S}(\mu, \nu)$ as a function of the lower order terms $w_{\mathbb{C}^{2}}^{[c d]}\left(\mu^{\prime}, \nu^{\prime}\right)$. Substituting this in, we arrive at a formula for $w_{\mathbb{C}^{2}}^{[a b]}(\mu, \nu)$ in lower order terms which also holds for $g_{\mathbb{C}^{2}}(\mu, \nu)$ whenever $\ell(\mu)>1$ or $\ell(\nu)>1$. The base cases $\mu=\emptyset, \nu=\emptyset$ are covered by Lehn's theorem, so what is left is to prove the case $\ell(\mu)=\ell(\nu)=1$.

\section{6}

In fact, by the induction step above, we only need to check that

$$
(\mathrm{W}(\mathcal{L}) \eta, \xi)=(\Gamma(\mathcal{L}) \eta, \xi)
$$

for an arbitrary pair of classes

$$
\xi \in H_{T}^{*}\left(\operatorname{Hilb}_{k} \mathbb{C}^{2}\right), \quad \eta \in H_{T}^{*}\left(\operatorname{Hilb}_{l} \mathbb{C}^{2}\right),
$$

having nonzero inner product with the classes $\alpha_{-k}|\rangle$ and $\alpha_{-l}|\rangle$, respectively.

To do this computation, we first need to set up the equivariant localization.

\section{7}

Let $T \cong\left(\mathbb{C}^{*}\right)^{2} \subset G L(2)$ be the standard maximal torus, i.e.

$$
\operatorname{Lie} T=\left\{\left(\begin{array}{ll}
t_{1} & \\
& t_{2}
\end{array}\right)\right\}
$$


In equivariant cohomology, we may identify $\mathcal{F}_{\mathbb{C}^{2}}$ with symmetric polynomials over $\mathbb{Q}\left[t_{1}, t_{2}\right]$ by requiring that |\rangle corresponds to 1 and

$$
\alpha_{-n}(1) \mapsto p_{n}
$$

that is, the Nakajima operators become operators of multiplication by powersum functions. It is known, see [15, 22] and also e.g. [21], that the classes of fixed points then correspond to properly normalized Jack polynomials.

Concretely, take a monomial ideal

$$
I_{\lambda}=\left(x_{1}^{\lambda_{i}} x_{2}^{i-1}\right) \subset \mathbb{C}\left[x_{1}, x_{2}\right]
$$

Then

$$
\left.\left[I_{\lambda}\right] \mapsto t_{2}^{|\lambda|} J_{\lambda}\right|_{p_{i}=t_{1} p_{i}}
$$

where $\left[I_{\lambda}\right]$ is the cohomological dual to the fixed point $I_{\lambda} \in \mathrm{Hilb}_{n} \mathbb{C}^{2}$, and $J_{\lambda}$ is the integral Jack polynomial with parameter

$$
\theta=-t_{2} / t_{1}
$$

The corresponding inner-product is

$$
\left(p_{\lambda}, p_{\mu}\right)_{t_{1}, t_{2}}=\delta_{\lambda, \mu} \frac{(-1)^{|\lambda|-\ell(\lambda)}}{\mathfrak{z}(\lambda) t_{1}^{\ell(\lambda)} t_{2}^{\ell(\lambda)}}
$$

where

$$
\mathfrak{z}(\lambda)=\frac{1}{\operatorname{aut}(\lambda) \prod_{i} \lambda_{i}}
$$

is the usual factor [16].

\section{8}

Our next goal is to compute the character of the $T$-module

$$
\left.\mathrm{E}\right|_{\left(I_{\lambda}, I_{\mu}\right)}=\chi(\mathcal{O}, \mathcal{O})-\chi\left(I_{\lambda}, I_{\mu}\right) .
$$

Given a $T$-module $V$ with finite-dimensional weight spaces, we denote by $[V]$ denote its character in $\mathbb{C}\left[\left[z_{1}, z_{1}^{-1}, z_{2}, z_{2}^{-1}\right]\right]$. Concretely, $[V]$ is represented by the trace of element $\left(z_{1}, z_{2}\right) \in T$ in its action on $V$. 


\section{Lemma 6.}

$$
\left[\left.\mathrm{E}\right|_{\left(I_{\lambda}, I_{\mu}\right)}\right]=\sum_{\square \in \lambda} z_{1}^{a_{\lambda}(\square)+1} z_{2}^{-l_{\mu}(\square)}+\sum_{\square \in \mu} z_{1}^{-a_{\mu}(\square)} z_{2}^{l_{\lambda}(\square)+1} .
$$

Proof. By Riemann-Roch, we have

$$
\chi\left(I_{\lambda}, I_{\mu}\right)=\frac{\left[I_{\lambda}^{\vee}\right]\left[I_{\mu}\right]}{\left[\mathcal{O}^{\vee}\right]}=\frac{\left[I_{\lambda}\right]^{\vee}\left[I_{\mu}\right]}{[\mathcal{O}]^{\vee}}
$$

where $f\left(z_{1}, z_{2}\right)^{\vee}=f\left(z_{1}^{-1}, z_{2}^{-1}\right)$. Substituting

$$
\left[I_{\mu}\right]=\sum_{i \geq 1} \frac{z_{1}^{-\mu_{i}} z_{2}^{1-i}}{1-z_{1}^{-1}}, \quad\left[I_{\lambda}\right]=\sum_{j \geq 1} \frac{z_{2}^{-\lambda_{j}^{\prime}} z_{1}^{1-j}}{1-z_{2}^{-1}}
$$

where $\lambda^{\prime}$ is the transposed diagram, into the above formula yields

$$
\left[\left.\mathrm{E}\right|_{\left(I_{\lambda}, I_{\mu}\right)}\right]=\sum_{i, j \geq 1} z_{1}^{j-\mu_{i}} z_{2}^{\lambda_{j}^{\prime}-i+1}-\sum_{i, j \geq 1} z_{1}^{j} z_{2}^{1-i} .
$$

Now observe the terms for which the exponent of $z_{1}$ is $\leq 0$ occur only in the first sum and correspond to the second sum in (23). For these terms then, the two formulas are equal. The remaining terms may be determined using Serre duality which implies

$$
\left[\left.\mathrm{E}\right|_{\left(I_{\lambda}, I_{\mu}\right)}\right]=z_{1} z_{2}\left[\left.\mathrm{E}\right|_{\left(I_{\mu}, I_{\lambda}\right)}\right]^{\vee}
$$

Note that the same argument with the roles of $z_{1}$ and $z_{2}$ interchanged yields the same formula except the ranges of summation $\square \in \lambda$ and $\square \in \mu$ are interchanged.

The Lemma shows that (6) for the equivariant cohomology of $\mathrm{Hilb}_{n} \mathbb{C}^{2}$ is equivalent to the Pieri-type formula (13).

We also note that the character is a sum of exactly $|\mu|+|\lambda|$ terms, implying the vanishing (15). 


\section{9}

It is well known that Jack polynomials labeled by single row or column have nonzero inner product with the power-sum function of the same degree (this is true already in the Schur function case $\theta=1$ ). Therefore, as we saw in Section 3.6, it suffices to prove (13) in the special case

$$
\lambda=\left(1^{k}\right), \quad \mu=(l) .
$$

Since multiplication by a function adds squares to diagrams and there are only two diagram fitting inside both $\lambda$ and $\mu$, we have

$$
\begin{aligned}
& \left(E^{m}\left(E^{*}\right)^{\theta-m-1} J_{\lambda}, J_{\mu}\right)_{\theta}= \\
& \left(E^{m}, J_{\mu}\right)_{\theta}\left(E^{\theta-m-1}, J_{\lambda}\right)_{\theta}+\theta\left(E^{m} p_{1}, J_{\mu}\right)_{\theta}\left(E^{\theta-m-1} p_{1}, J_{\lambda}\right)_{\theta}
\end{aligned}
$$

the factor of $\theta$ in the second term coming from $\left(p_{1}, p_{1}\right)_{\theta}^{-1}=\theta$.

We have

$$
\left(E^{m}, J_{\mu}\right)_{\theta}=\theta^{-l} \prod_{i=0}^{l-1}(m-i), \quad\left(E^{m} p_{1}, J_{\mu}\right)_{\theta}=l \theta^{-l} \prod_{i=0}^{l-2}(m-i)
$$

which is an elementary statement about polynomials in one variable. Dually,

$$
\left(E^{m}, J_{\lambda}\right)_{\theta}=\theta^{-k} \prod_{i=0}^{k-1}(m+i \theta), \quad\left(E^{m} p_{1}, J_{\lambda}\right)_{\theta}=l \theta^{-k} \prod_{i=0}^{k-2}(m+i \theta) .
$$

Replacing $m$ by $\theta-1-m$ in the above formula and summing up gives the right answer. This concludes the proof.

\section{References}

[1] D. Bradley, Multiple q-zeta values, Journal of Algebra (2005), 283, 752 $-798$.

[2] M. Brion, Equivariant cohomology and equivariant intersection theory Representation theory and algebraic geometry, Kluwer (1998), pp. 1-37

[3] E. Carlsson, Vertex Operators and Moduli Spaces of Sheaves, PhD Thesis, Princeton University, arXiv:0906.1825. 
[4] E. Carlsson, N. Nekrasov, A. Okounkov On vertex operators in gauge theories, to appear.

[5] N. Chriss, V. Ginzburg, Representation Theory and Complex Geometry, Birkhauser, Boston (1997).

[6] D. Edidin, W. Graham, Equivariant Intersection theory, Invent. Math v. 131 (1996), $595-634$

[7] G. Ellingsrud, L. Göttsche, M. Lehn, On the cobordism class of the Hilbert scheme of a surface Journal of Algebraic Geometry, 10 (2001), $81-100$.

[8] W. Fulton, Equivariant cohomology in algebraic geometry, http://www.math.washington.edu/ dandersn/eilenberg/

[9] L. Göttsche, Hilbert schemes of points on surfaces, ICM Proceedings, Vol. II (Beijing, 2002), 483-494.

[10] I. Grojnowski, Instantons and affine algebras I: the Hilbert scheme and vertex operators, Math. Res. Lett. 3 (1996), 275-291.

[11] V. Guillemin, S. Sternberg, Supersymmetry and Equivariant de Rham Theory, Springer-Verlag (1999).

[12] M. Lehn, Chern Classes of Taulogical Sheaves on Hilbert Schemes of Points on Surfaces, Inventiones Math., 136 (1999) no. 1, 157-207.

[13] M. Lehn, Geometry of Hilbert schemes, CRM Proceedings and Lecture Notes, Volume 38, 2004, 1 - 30.

[14] W. Li, Z. Qin, W. Wang, Vertex algebras and the cohomology ring structure of Hilbert schemes of points on surfaces, Math. Ann. 324 (2002), 105-133.

[15] W. Li, Z. Qin, W. Wang, The cohomology rings of Hilbert schemes via Jack polynomials, CRM Proceedings and Lecture Notes, vol. 38 (2004), 249-258.

[16] I. Macdonald, Symmetric functions and Hall polynomials, The Clarendon Press, Oxford University Press, New York, 1995. 
[17] D. Maulik and A. Okounkov, Quantum cohomology of framed sheaves, to appear.

[18] H. Nakajima, Heisenberg algebra and Hilbert schemes of points on projective surfaces, Ann. of Math. (2) 145 (1997), no. 2, 379-388.

[19] H. Nakajima, Lectures on Hilbert schemes of points on surfaces, AMS, Providence, RI, 1999.

[20] N. Nekrasov and A. Okounkov, Seiberg-Witten Theory and Random Partitions, In The Unity of Mathematics (ed. by P. Etingof, V. Retakh, I. M. Singer) Progress in Mathematics, Vol. 244, Birkhäuser, 2006, hep-th/0306238.

[21] A. Okounkov and R. Pandharipande, Quantum cohomology of the Hilbert scheme of points in the plane, arXiv:math/0411210.

[22] E. Vasserot, Sur l'anneau de cohomologie du schéma de Hilbert de $\mathbf{C}^{2}$, C. R. Acad. Sci. Paris Sér. I Math. 332 (2001), no. 1, 7-12. 